Research Article

\title{
Steady-State Dynamical Response of a Strongly Nonlinear System with Impact and Coulomb Friction Subjected to Gaussian White Noise Excitation
}

\author{
Guidong Yang $\mathbb{D}^{1},{ }^{1}$ Dongmei Huang, ${ }^{1}$ Wei Li, ${ }^{1}$ and Meng Su${ }^{2}$ \\ ${ }^{1}$ School of Mathematics and Statistics, Xidian University, No. 2 South Taibai Road, Xi'an, Shaanxi 710071, China \\ ${ }^{2}$ Department of Applied Mathematics, Northwestern Polytechnical University, Xi'an, Shaanxi 710072, China \\ Correspondence should be addressed to Guidong Yang; gdyang@xidian.edu.cn
}

Received 12 April 2020; Revised 30 July 2020; Accepted 17 September 2020; Published 28 September 2020

Academic Editor: Francesco Pellicano

Copyright (c) 2020 Guidong Yang et al. This is an open access article distributed under the Creative Commons Attribution License, which permits unrestricted use, distribution, and reproduction in any medium, provided the original work is properly cited.

\begin{abstract}
The paper is devoted to the steady-state dynamical response analysis of a strongly nonlinear system with impact and Coulomb friction subjected to Gaussian white noise excitation. The Zhuravlev nonsmooth transformation of the state variables combined with the Dirac delta function is utilized to simplify the original system to one without velocity jump. Then, the steady-state probability density functions of the transformed system are derived in terms of the stochastic averaging method of energy envelope. The effectiveness of the presented analytical procedure is verified by those from the Monte Carlo simulation based on the original system. Effects of different restitution coefficients, amplitudes of friction, and noise intensities on the steady-state dynamical responses are investigated in detail. Results show different intensities of Gaussian white noise can affect the peaks value of the probability density functions, whereas the variations of restitution coefficients and amplitudes of friction can induce the occurrence of stochastic P-bifurcation.
\end{abstract}

\section{Introduction}

Nowadays, mechanical systems have become more and more intricate due to the rapid development of science and technology [1-4]. Impact and friction, as two types of inevitable nonsmooth factors in engineering and structural applications, may change the dynamical properties of mechanical systems significantly and even result in the structural insecurity [5]. On the one hand, gaps are widely distributed in the components of mechanical equipment as a result of manufacturing errors. They may bring about collision and friction among different components, which can cause noises and reduce the working efficiency of the devices [6]. On the other hand, engineering structures usually work in complex external environment and are inevitably affected by random excitations such as ground motion, atmospheric turbulence, wind, and road unevenness. [7-9]. The random excitations owing to impact and friction or environment may also play important roles in responses of mechanical systems $[10,11]$. Hence, it is of great urgency and cardinal significance to study the dynamical behaviors of systems with impact and friction under random excitations.

As systems with impact and friction are notorious for their inherent nonsmooth features which lead to the difficulties of analytical study $[12,13]$, a large number of experts and scholars at home and abroad have to appeal to the numerical investigation. Accordingly, abundant achievements are gained about chaotic motions, Hopf bifurcations, and subharmonic oscillation [14-17]. Furthermore, some particular dynamical phenomena are observed such as grazing bifurcation, chatter, and sticking motions [18-20]. In terms of the mean Poincaré mapping, Feng and He [21] investigated the stochastic stick-slip motion of a random friction model. With the help of the generalized cell mapping method, Sun [22] studied random response problems on systems with Coulomb friction. Virgin and Begley [23] showed the basin of attraction due to friction and impact of a vibro-impact system with Coulomb friction damping based 
on the global dynamical analysis. Zhang et al. [24] discussed the evolution process to the chaos of a single-degree-offreedom vibro-impact system while Yue et al. [25] focused on the coexistence of strange nonchaotic attractors and a novel mixed attractor in a periodically driven three-degreeof-freedom vibro-impact system with symmetry.

In recent years, more and more attention has been paid to the analytical study of the nonsmooth system. Several different models were established for impact system and frictional systems separately to make the response analysis tractable. For systems with impact, one relatively easy model was based on the Hertzian contact law. The response's closed-form solutions were presented by Jing and Sheu [26] for a single-degree-of-freedom vibro-impact system. Using the stochastic averaging technique, Huang et al. [27] obtained the stationary responses of a multi-degree-of-freedom vibro-impact system with clearance. To overcome the disadvantage that the Hertzian contact model is impotent for dissipated contact problem, $\mathrm{Xu}$ et al. [28] developed a modified Hertzian contact model and analysed random vibration problems of inelastic vibro-impact systems. Another impact model commonly used was the classical impact which could depict the energy loss but was not easy to obtain the analytical solutions of responses. A nonsmooth coordinate transformation contributed to Zhuravlev [29] was introduced to transform the vibro-impact system to one without velocity jump. By this transformation, some analytical methods applicable to smooth systems were extended to the vibro-impact ones. By means of the stochastic averaging method and energy balance method, Dimentberg et al. [30-32] investigated the response probability density function, energy loss, and first passage problem of the "pseudolinear" vibro-impact system. Zhu [33] obtained one solution of stationary response for the single-degree-of-freedom vibro-impact system by the exponential-polynomial closure method. With the help of Zhuravlev-Ivanov transformation and the iterative method of weighted residue, Chen et al. [34] discussed the closed-form stationary probability distribution for stochastically excited vibro-impact oscillators. Based on the framework of Galerkin technique, Xie et al. [35] studied the transient response of a vibro-impact system under random excitation. For frictional systems, more than ten models were developed for different cases. The most commonly used one was the Coulomb friction model. Ding [36] discussed the calculation for an ideal dry friction system. Sun et al. [37] investigated the reliability of a nonlinear damped friction oscillator under combined additive and multiplicative Gaussian white noise excitations by the stochastic averaging method and obtained satisfactory results.

Although the problems of the vibro-impact system and friction system have been studied by many experts and scholars with different methods, most of them are limited to separate research. The analytical studies for the vibro-impact system with friction under random excitations are far from enough and there are numerous problems need to be solved. Recently, Su et al. [38] combined the nonsmooth coordinate transformation and the stochastic method of the amplitude to study the stationary responses of a weakly nonlinear system with impact and Coulomb friction under Gaussian white noise. The proposed method was quite effective. However, in the case of strongly nonlinear restoring force, the stochastic method of the amplitude may be invalid to obtain accurate results. To deal with this problem, the research is performed in this paper, and the layout is as follows. The problems for a strongly nonlinear system with impact and Coulomb friction subjected to Gaussian white noise excitation are formulated in Section 2. The approximately equivalent system is obtained by the nonsmooth coordinate transformation. In Section 3, the steady-state responses' probability density functions are derived analytically by means of the stochastic averaging method of energy envelope. The effectiveness of the proposed method is verified in Section 4. Additionally, in this section, the influences of impact, friction, and random excitation on the system's responses are discussed in detail. At last, the paper is ended with conclusions.

\section{Problem Formulation}

Consider a randomly excited single-degree-of-freedom Van der Pol-Duffing vibro-impact system with Coulomb friction whose schematic model is displayed in Figure 1. The motion of the system is governed by

$$
\ddot{x}+\varepsilon\left(c_{2} x^{2}-c_{1}\right) \dot{x}+\varepsilon f_{k} \operatorname{sgn}(\dot{x})+x^{3}=\varepsilon^{1 / 2} \xi(t), \quad x>0,
$$

$$
\dot{x}_{+}=-r \dot{x}_{-}, \quad x=0,
$$

where $\varepsilon$ is a small positive number used to limit the scale of other parameters, $c_{2}$ and $c_{1}$ are the nonlinear and linear damping coefficients, respectively, $f_{k}$ indicates the amplitude of friction and $\operatorname{sgn}(\dot{x})$ represents the signum function with respect to the velocity $x, \xi(t)$ denotes a Gaussian white noise process with zero mean and correlation function $R\left(t_{2}-t_{1}\right)=2 D \delta\left(t_{2}-t_{1}\right)$, in which $\delta(\bullet)$ is the Dirac delta function, meaning that the noise intensity is $2 D, \dot{x}_{-}$and $\dot{x}_{+}$ refer to values of velocity at the instants right before and after the impact, respectively, $r$ is the impact restitution coefficient satisfying $0<r \leq 1$, which depicts the energy loss due to impact, and $x=0$ describes the impact condition, that is, when the oscillator arrives at the position $x=0$, the impact happens.

From equations (1) and (2), the motion of the oscillator is performed in two steps. The oscillator moves freely between two consecutive impacts. Then, its velocity changes suddenly at the static equilibrium position of the system. Besides, the dry friction force also has a discontinuous variety in terms of the direction of velocity. Thus, the vibroimpact system with dry friction is strongly nonlinear as well as nonsmooth.

To overcome the difficulty due to the nonsmooth characteristics of the vibro-impact system, the nonsmooth variable transformation proposed by Zhuravlev [29] is introduced as follows: 


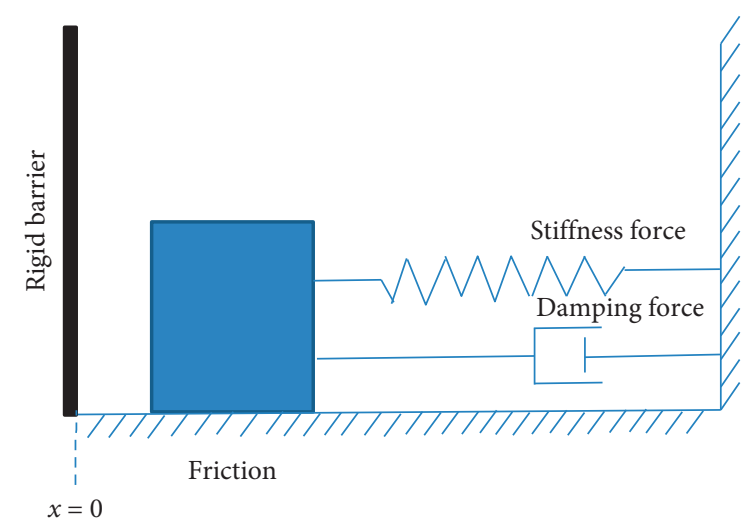

Figure 1: Schematic model of the system with impact and friction.

$$
\begin{aligned}
& x=x_{1}=|y|, \\
& \dot{x}=x_{2}=\dot{y} \operatorname{sgn} y, \\
& \ddot{x}=\ddot{y} \operatorname{sgn} y,
\end{aligned}
$$

in which

$$
\operatorname{sgn} y= \begin{cases}1, & y>0, \\ -1, & y<0 .\end{cases}
$$

Obviously, the original phase plane $(x, \dot{x})$ is mapped to the whole phase plane $(y, \dot{y})$ by the nonsmooth variable transformation, in which the restrictive condition $x>0$ is removed.

Substituting equation (3) into equations (1) and (2) results in the following expressions:

$$
\ddot{y}+\varepsilon\left(c_{2} y^{2}-c_{1}\right) \dot{y}+\varepsilon f_{k} \operatorname{sgn}(\dot{y})+y^{3}=\varepsilon^{1 / 2} \operatorname{sgn} y \xi(t), \quad t \neq t_{*},
$$

$$
\begin{aligned}
\ddot{y}_{+} & =r \dot{y}_{-}, \\
t & =t_{*},
\end{aligned}
$$

where $t=t_{*}$ indicates the time when impact occurs, corresponding to $x=0$. Comparing equation (6) with equation (2), it is easy to see that the velocity jump of the original variable at the rigid barrier is $\Delta \dot{x}=\dot{x}_{+}-\dot{x}_{-}=(-r-1) \dot{x}_{-}$, whereas the velocity jump for new variable is $\Delta \dot{y}=\dot{y}_{+}-\dot{y}_{-}=(r-1) \dot{y}_{-}$. The closer $r$ is to one, the smaller the velocity jump of the new variable is. In terms of the properties of the Dirac delta function, the following equation could be derived:

$$
\left(\dot{y}_{-}-\dot{y}_{+}\right) \delta\left(t-t_{*}\right)=(1-r) \dot{y}|\dot{y}| \delta(y)
$$

which can be combined into equation (5) as an additional impulsive term. Arranging them yields

$$
\begin{aligned}
\ddot{y} & +\varepsilon\left(c_{2} y^{2}-c_{1}\right) \dot{y}+\varepsilon f_{k} \operatorname{sgn}(\dot{y})-(r-1) \dot{y}|\dot{y}| \delta(y) \\
& +y^{3}=\varepsilon^{1 / 2} \operatorname{sgn} y \xi(t) .
\end{aligned}
$$

It is obvious that the new equation does not include the velocity jump, which may permit us to study it by taking advantage of some analytical methods applicable to the smooth system.

\section{Stochastic Averaging Method of Energy Envelope}

Considering the case that the energy change is caused by the linear damping, the nonlinear damping, the impact, and friction as well as random excitation is far less than the original energy of the system. Herein, the system could be quasiconservative. Thus, the stochastic averaging method of energy envelope may be feasible to deal with the approximately equivalent system described by equation (8).

The corresponding undamped system of equation (8) is

$$
\ddot{y}+y^{3}=0 .
$$

Letting $y=y_{1}, \dot{y}=y_{2}, g\left(y_{1}\right)=y_{1}^{3}$, the energy envelope of the system is written as

$$
\begin{array}{r}
H=\frac{1}{2} y_{2}^{2}+G\left(y_{1}\right), \\
G\left(y_{1}\right)=\int_{0}^{y_{1}} g(u) \mathrm{d} u,
\end{array}
$$

where $G\left(y_{1}\right)$ is the potential energy.

Equation (8) is equivalent to the following set of Itô stochastic differential equations:

$$
\begin{aligned}
& \mathrm{d} y_{1}=y_{2} \mathrm{~d} t, \\
& \mathrm{~d} y_{2}=\left[-f\left(y_{1}, y_{2}\right)-g\left(y_{1}\right)\right] \mathrm{d} t+\sigma\left(y_{1}, y_{2}\right) \mathrm{d} W(t),
\end{aligned}
$$

in which $\quad f\left(y_{1}, y_{2}\right)=\varepsilon\left(c_{2} y_{1}^{2}-c_{1}\right) y_{2}+\varepsilon f_{k}$ sgn $\left(y_{2}\right)-(r-1) y_{2}\left|y_{2}\right| \delta\left(y_{1}\right)$ represents the effective damping, $g\left(y_{1}\right)$ is the effective conservative force, $-f\left(y_{1}, y_{2}\right)-g\left(y_{1}\right)$ is the drift coefficient and $\sigma\left(y_{1}, y_{2}\right)$ is called the diffusion coefficient, and $W(t)$ denotes the Wiener process.

The Itô stochastic differential equations for the displacement $y_{1}$ and velocity $y_{2}$ can be transformed into the following Itô equations for displacement $y_{1}$ and the energy envelope $H(t)$ by the transformation equation (10) and the Itô's differential rule.

$$
\begin{aligned}
\mathrm{dy}_{1}= & \pm \sqrt{2 H-2 G\left(y_{1}\right)} \mathrm{d} t \\
\mathrm{~d} H=[ & \mp \varepsilon^{2} \sqrt{2 H-2 G\left(y_{1}\right)} f\left(y_{1}, \pm \sqrt{2 H-2 G\left(y_{1}\right)}\right) \\
& \left.+\frac{1}{2} \sigma^{2}\left(y_{1}, \pm \sqrt{2 H-2 G\left(y_{1}\right)}\right)\right] \mathrm{d} t \\
& \pm \sqrt{2 H-2 G\left(y_{1}\right)} \sigma\left(y_{1}, \pm \sqrt{2 H-2 G\left(y_{1}\right)}\right) \mathrm{d} W(t) .
\end{aligned}
$$

Noting that the displacement process $y_{1}$ is rapidly varying and the energy envelope process $H$ is a slowly varying one, according to the Khasminskii theorem [39], the $H$ process converges weakly to a one-dimensional Markov process as $\varepsilon \longrightarrow 0$, in a time interval $0<t<T$, where $T \sim o(1 / \varepsilon)$. Here, we also use the symbol $H$ to represent this one-dimensional Markov process. 
Averaging to equation (12), the resulting Itô equation is given by

$$
\mathrm{d} H=U(H) \mathrm{d} t+V(H) \mathrm{d} W(t),
$$

in which

$$
\begin{aligned}
T= & 4 T_{1 / 4}=4 \int_{0}^{A} \frac{\mathrm{d} y}{\sqrt{2 H-1 / 2 y_{1}^{4}}}=\sqrt{\pi} H^{-1 / 4} \frac{\Gamma(1 / 4)}{\Gamma(3 / 4)}, \\
U(H)= & -\frac{32}{5} \varepsilon c_{2} \frac{\Gamma^{2}(3 / 4)}{\Gamma^{2}(1 / 4)} H^{3 / 2}+\frac{4}{3} \varepsilon c_{1} H-\frac{4 \sqrt[4]{4}}{\sqrt{\pi}} \varepsilon f_{k} \frac{\Gamma(3 / 4)}{\Gamma(1 / 4)} H^{1 / 2} \\
& +\frac{4}{\sqrt{\pi}}(r-1) \frac{\Gamma(3 / 4)}{\Gamma(1 / 4)} H^{5 / 4}+\varepsilon D, \\
V^{2}(H)= & \frac{8}{3} \varepsilon D H,
\end{aligned}
$$

where $T$ is a pseudoperiod, $A$ denotes the amplitude, and $U(H) V^{2}(H)$ represent the averaged drift coefficient and diffusion coefficient, respectively.

During the calculation process, some points may be noted. Firstly, the averaging to the impact damping term should be done in half pseudoperiod since there are two impacts during this time. Secondly, the following formula is used to simplify the integral calculation:

$$
\int_{0}^{\pi / 2} \sin ^{m-1} \theta \cos ^{n-1} \theta \mathrm{d} \theta=\frac{1}{2} B\left(\frac{m}{2}, \frac{n}{2}\right)
$$

where $B(\cdot, \cdot)$ is the beta function.

The Fokker-Planck-Kolmogorov (FPK) equation corresponding to Itô equation (13) is easy to obtain as

$$
\frac{\partial p}{\partial t}=-\frac{\partial}{\partial H}[u(H) p]+\frac{1}{2} \frac{\partial^{2}}{\partial H^{2}}\left[v^{2}(H) p\right],
$$

in which the boundary condition is

$$
\left\{\begin{array}{l}
0 \leq p<\infty, \\
H=0, \\
p \longrightarrow 0, \\
\frac{\mathrm{d} p}{\mathrm{~d} H} \longrightarrow 0, \\
H \longrightarrow \infty .
\end{array}\right.
$$

The instantaneous response of equation (17) is usually difficult to obtain due to the existence of strongly nonlinear factors, although some special cases could be solved by the Galerkin procedure. We herein study the stationary dynamical response analytically.

Taking the left side of equation (17) to be zero and integrating at both sides, the stationary probability density function of energy envelope $H$ is deduced as

$$
p(H)=\frac{C}{K} H^{-1 / 4} \exp \left(\mathrm{MH}^{3 / 2}+\mathrm{NH}+\mathrm{OH}^{1 / 2}+\mathrm{QH}^{5 / 4}\right)
$$

in which $C$ is a normalization constant and

$$
\begin{aligned}
& K=\frac{8}{3} \varepsilon D, \\
& M=-\frac{16 c_{2}}{5 D} \frac{\Gamma^{2}(3 / 4)}{\Gamma^{2}(1 / 4)}, \\
& N=\frac{c_{1}}{D}, \\
& O=\frac{-6 \sqrt{2} f_{k}}{\sqrt{\pi} D} \frac{\Gamma(3 / 4)}{\Gamma(1 / 4)}, \\
& Q=\frac{12(r-1)}{5 \sqrt{\pi} \varepsilon D} \frac{\Gamma(3 / 4)}{\Gamma(1 / 4)} .
\end{aligned}
$$

As is shown by Stratonovitch [40], the corresponding first approximation for the joint probability density function of the displacement $y_{1}$ and velocity $y_{2}$ can be obtained as follows in terms of equations (7) and (14):

$$
\left.p\left(y_{1}, y_{2}\right) \simeq \frac{p(H)}{T(H)}\right|_{H=(1 / 2) y_{2}^{2}+(1 / 4) y_{1}^{2}} .
$$

Note that $g\left(y_{1}\right)=y_{1}^{3}$ has only one zero, then the transformation from $p(H)$ to $p\left(y_{1}, y_{2}\right)$ is one-to-one for each level of $H$ [41].

Since the displacement $y_{1}$ and velocity $y_{2}$ are the variables of the transformed system, the original system's joint probability density function for the displacement $x_{1}$ and velocity $x_{2}$ can be derived by means of the inverse transformation of equation (2).

$$
\begin{aligned}
p\left(x_{1}, x_{2}\right)= & p_{s}\left(y_{1}, y_{2}\right)\left|\frac{\partial\left(y_{1}, y_{2}\right)}{\partial\left(x_{1}, x_{2}\right)}\right|=p_{Y_{1}, Y_{2}}\left(x_{1}, x_{2}\right) \\
& +p_{Y_{1}, Y_{2}}\left(-x_{1},-x_{2}\right)=2 p\left(y_{1}, y_{2}\right) .
\end{aligned}
$$

According to the formula between the joint probability density function and the marginal probability density function, the resulting stationary probability density function for displacement $x_{1}$ and velocity $x_{2}$ are given by

$$
\begin{aligned}
& p_{X_{1}}\left(x_{1}\right)=\int_{R} p\left(x_{1}, s\right) \mathrm{d} s, \\
& p_{X_{2}}\left(x_{2}\right)=\int_{R^{+}} p\left(s, x_{2}\right) \mathrm{d} s .
\end{aligned}
$$

\section{Response Analysis}

In order to validate the analytical results of the system's stationary responses obtained via the nonsmooth transformation and stochastic averaging method of energy envelope, numerical simulations by fourth-order stochastic Runge-Kutta algorithm and Monte Carlo methods are performed, and herein the procedure is based on the original 
vibro-impact system with dry friction under white noise excitation. To judge the instants of impacts more accurately, two different time step sizes are used in the Runge-Kutta algorithm: before the oscillator moves to the barrier, the larger time step is chosen to reduce simulation time; once the oscillator arrives at the position $x=0$, the smaller time step is selected to improve the precision. Seeing the stochastic response, we are interested in is the stationary one, the frontal data should not be considered.

4.1. Effectiveness of the Procedure. In this subsection, the agreement between the analytical results and numerical results is under consideration. Fixing the scale parameter $\varepsilon=0.01$, since the analytical results are derived in the case that the energy loss due to the linear damping, the nonlinear damping, the impact, and friction as well as random excitation is far less than the system's original energy. Other parameters are as follows: $c_{1}=3.0, c_{2}=1.0, f_{k}=1.0$, $r=0.99$, and $D=1.0$. Figure 2 presents the analytical results and Monte Carlo simulation results of stationary PDFs for total energy $H$, where the line denotes the analytical results and the bullet means the numerical results. By comparing them with each other, excellent agreement can be found easily. The stationary joint PDFs for displacement $x_{1}$ and velocity $x_{2}$ are shown in Figure 3. It is obvious that the analytical result in Figure 3(a) agrees well with the Monte Carlo result in Figure 3(b). To reveal the effectiveness of the analytical procedure more clearly, the contour plots of joint PDFs corresponding to Figures 3(a) and 3(b) are depicted in Figures 4(a) and 4(b), respectively. The error is inconspicuous between the analytical result and Monte Carlo simulation result.

4.2. The Influence of Nonsmooth Factors. In virtue of the nonsmooth characteristics caused by impact and dry friction, the dynamical behaviour of the system becomes quite complex. Hence, the influences of nonsmooth factors on the system's responses would be investigated in detail.

First of all, we consider the changes of system response when the restitution coefficient $r$ varies as well as other system parameters keep unchanged. As mentioned above, the value of restitution coefficient $r$ depicts the energy loss due to impact. The closer $r$ is to 1 , the less energy loss is. Here, $r>0.9$ is considered to make the system quasiconservative, and thus, the analytical results are effective. The curves of stationary PDFs for total energy are shown in Figure 5 as the restitution coefficient $r$ equals to 0.95 , and herein other parameters remain unchanged. The line represents the analytical results and the bullet denotes the numerical results. It is obvious that they agree well with each other. The peak of the PDFs for total energy is at the position $H=0$ in Figure 5, that is, the oscillator would stay at the system's static equilibrium with a high probability. However, it is not the case shown in Figure 2, where $r$ equals to 0.99. It is intuitively believable that the smaller $r$ is, the larger energy loss is; consequently, the probability that the oscillator stays at the system's static equilibrium is higher. On the other hand, the results indicate the occurrence of stochastic

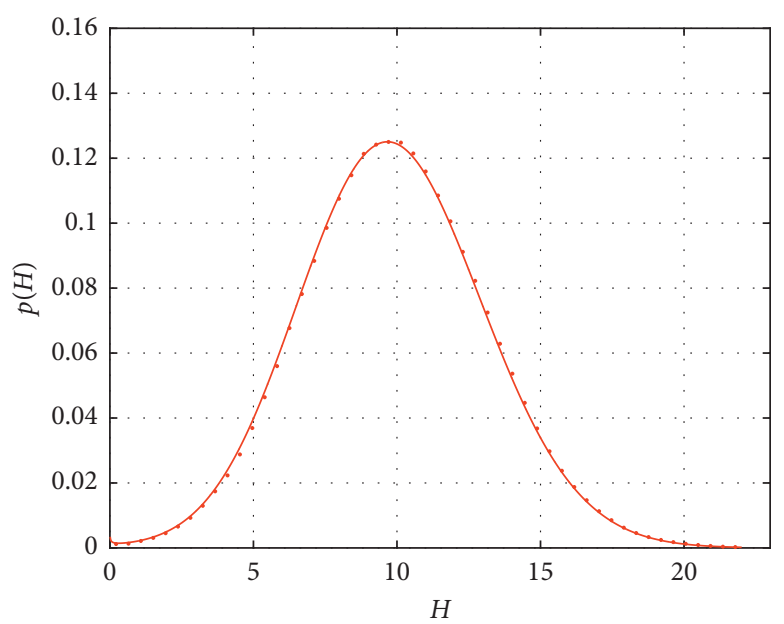

FIgURE 2: Stationary PDFs for total energy H. (-), analytical results; $(\cdot)$, Monte Carlo results.

P-bifurcation. In order to present this phenomenon more clearly, Figures 6(a) and 6(b) show the analytical results and numerical results of stationary joint PDFs for displacement $x_{1}$ and velocity $x_{2}$, respectively, as $r=0.95$. Also, Figures 7(a) and 7(b) are the corresponding contour plots. It is easy to find that the stochastic P-bifurcation happens by comparing Figures 6 and 7 with Figures 3 and 4, respectively, since the image in Figure 3 presents the shape of the crater while there is a singular peak in Figure 6. Furthermore, the section graphs of joint PDFs on the surface $x_{1}=0$ for different restitution coefficients $r$ are presented in Figure 8, who clearly demonstrate the evolution process of PDFs' images with $r$ varying. When $r=0.95$, the PDF only has one peak. Then, it peaks to three peaks as $r$ increases and finally becomes two peaks. This phenomenon is indicative of stochastic P-bifurcation, that is, the change of the restitution coefficient $r$ can induce the occurrence of stochastic P-bifurcation.

The dry friction is another significant nonsmooth factor which may have a great influence on the dynamical properties of the system. Therefore, we explore the changes of stationary joint PDFs for displacement $x_{1}$ and velocity $x_{2}$ when the amplitude of Coulomb friction $f_{k}$ varies. For this purpose, we fix other parameters as $c_{1}=3.0, c_{2}=1.0$, $r=0.99$, and $D=1.0$ and only modify the value of $f_{k}$. Analytical results of stationary joint PDFs for displacement $x_{1}$ and velocity $x_{2}$ for different amplitudes of friction are shown in Figure 9. It can be seen from Figure 9(a), the image of the system's response presents the shape of the crater when $f_{k}=0$, that is, there is no friction. The result with $f_{k}=2$ in Figure 9(b) is different from the case of $f_{k}=0$ in Figure 9(a), since at this time, a new peak arises. As for the case $f_{k}=2.5$ in Figure 9(c), the new peak becomes higher, and two other peaks become quite lower, and when $f_{k}=3$, there is only one singular peak in Figure 9(d). It is obvious that stochastic P-bifurcation happens as the amplitude of Coulomb friction varies. By the way of understanding the evolution process of stochastic P-bifurcation, the section graphs of joint PDFs on the surface $x_{1}=0$ for different 


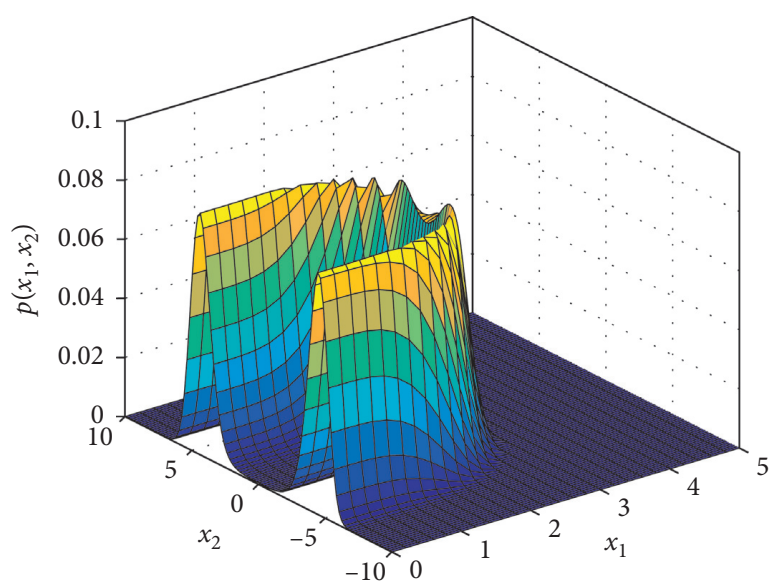

(a)

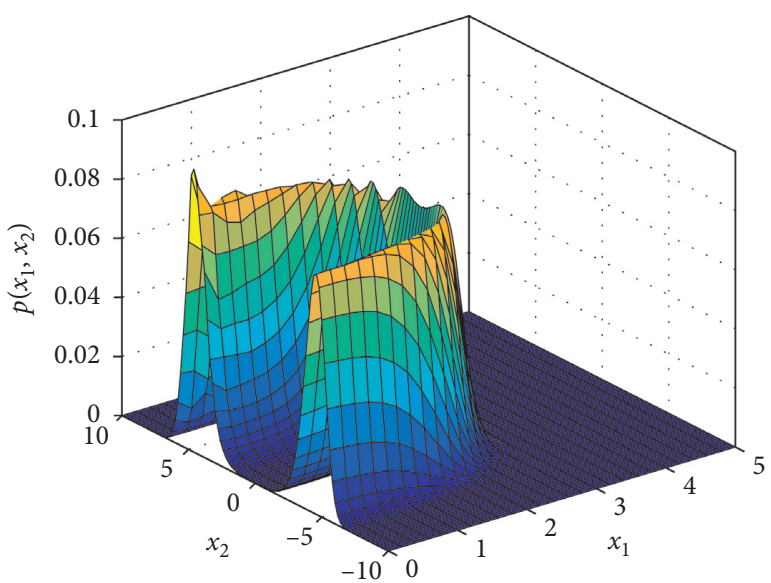

(b)

FIgURE 3: Stationary joint PDFs for displacement $x_{1}$ and velocity $x_{2}$ : (a) analytical results; (b) Monte Carlo results.

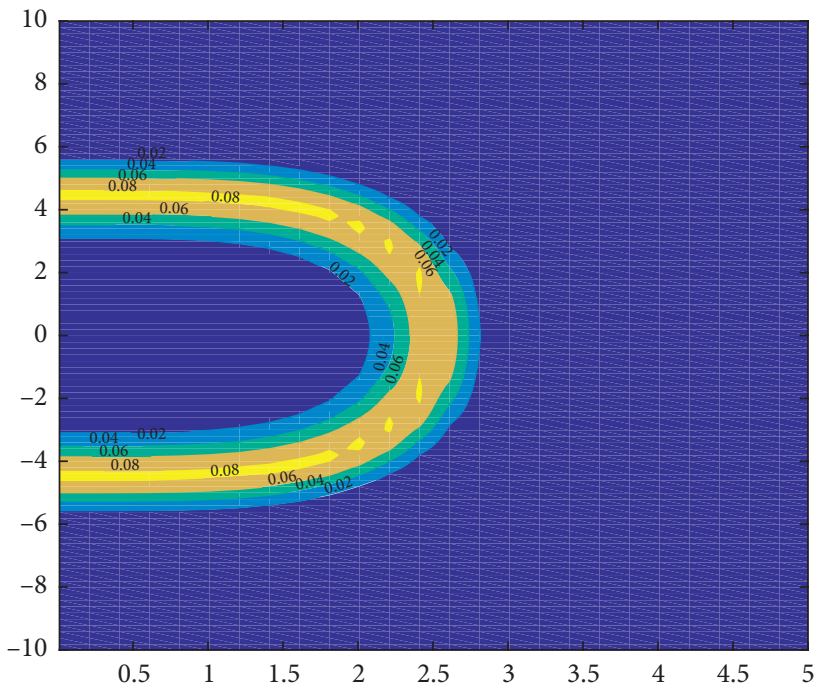

(a)

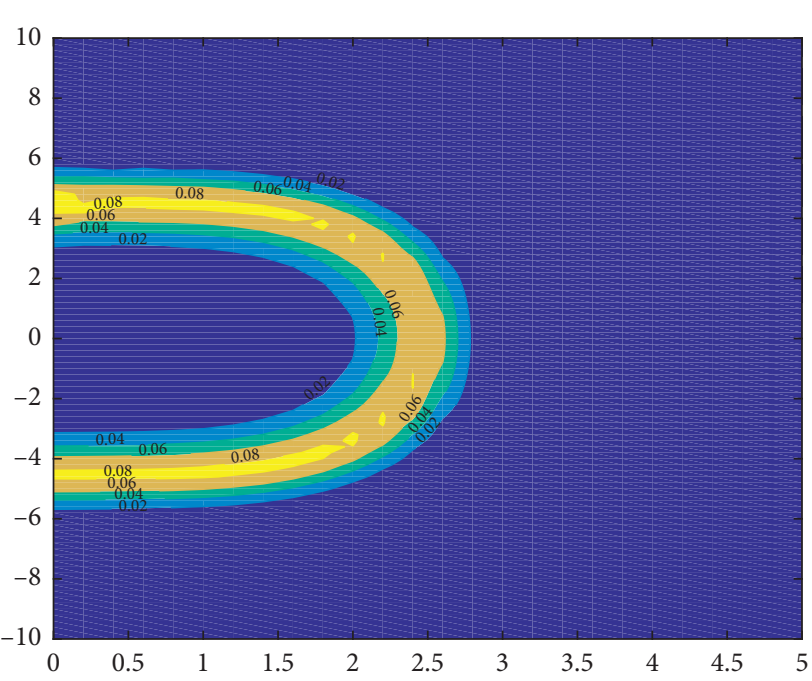

(b)

FIGURE 4: Contour plots of stationary joint PDFs for displacement $x_{1}$ and velocity $x_{2}$ : (a) analytical results; (b) Monte Carlo results.

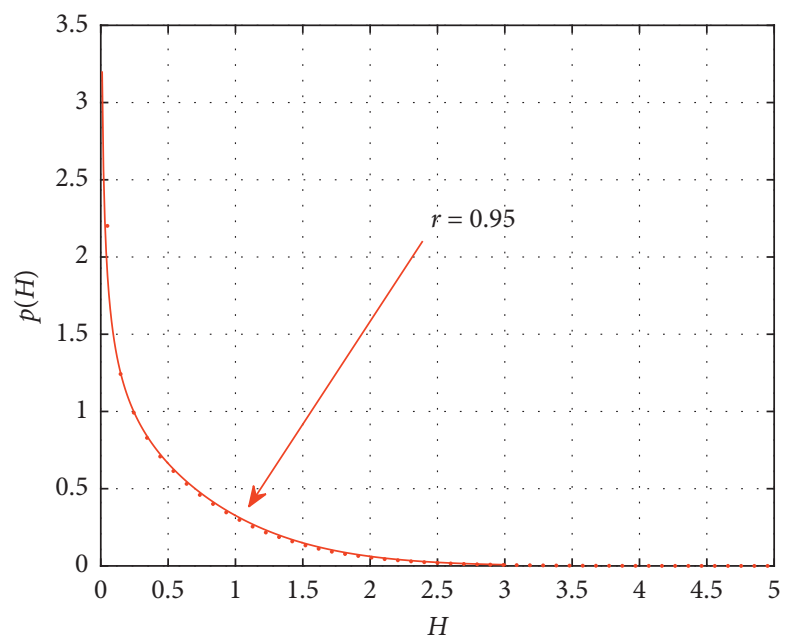

Figure 5: Stationary PDFs for total energy H. (-), analytical results; (.), Monte Carlo results. 


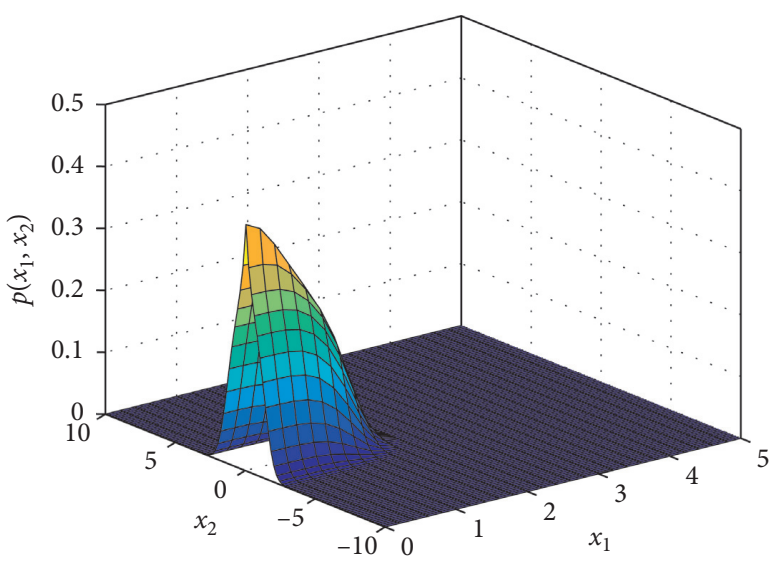

(a)

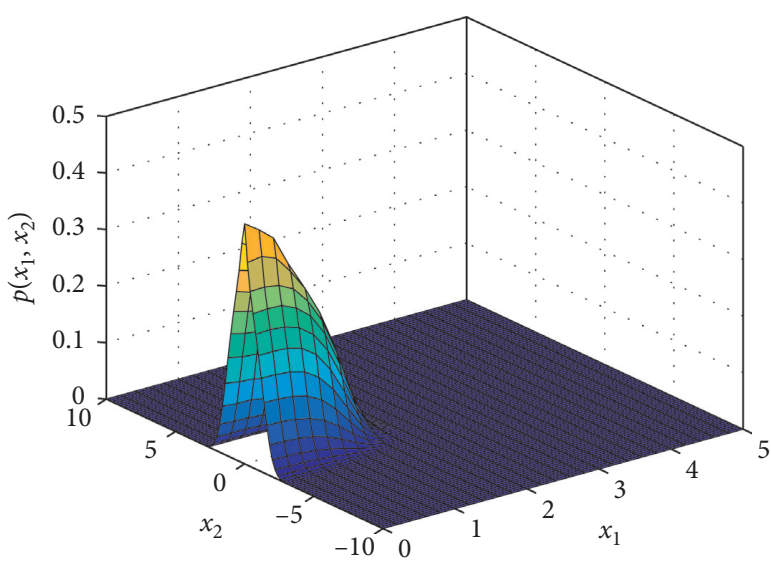

(b)

FIGURE 6: Stationary joint PDFs for displacement $x_{1}$ and velocity $x_{2}$ as $r=0.95$ : (a) analytical results; (b) Monte Carlo results.

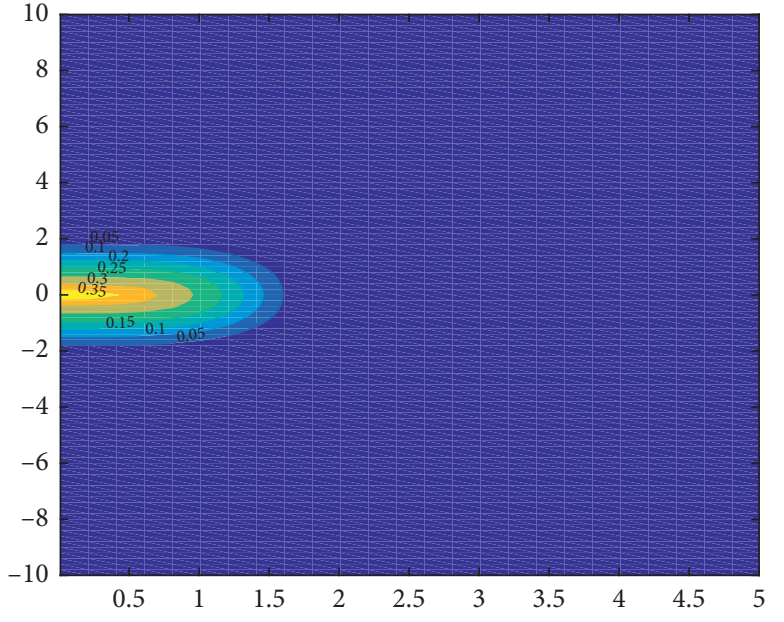

(a)

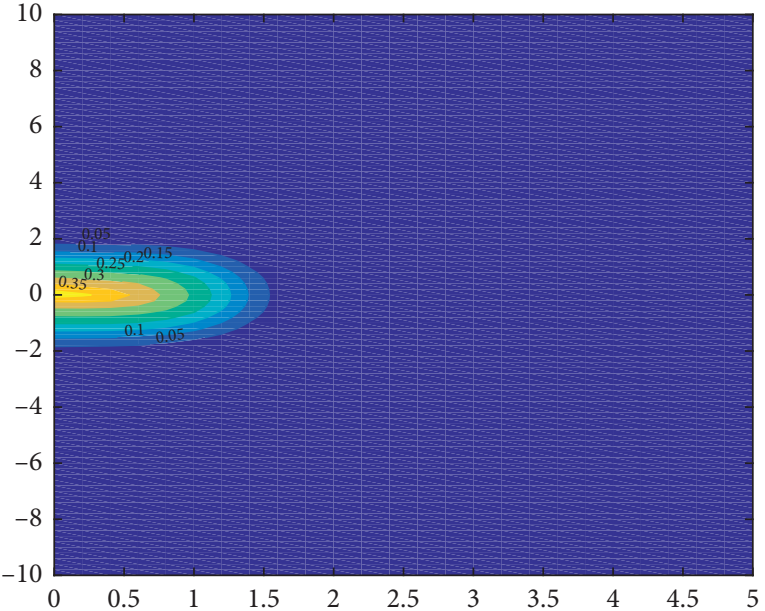

(b)

FIgUre 7: Contour plots of stationary joint PDFs for displacement $x_{1}$ and velocity $x_{2}$ as $r=0.95$ : (a) analytical results; (b) Monte Carlo results.

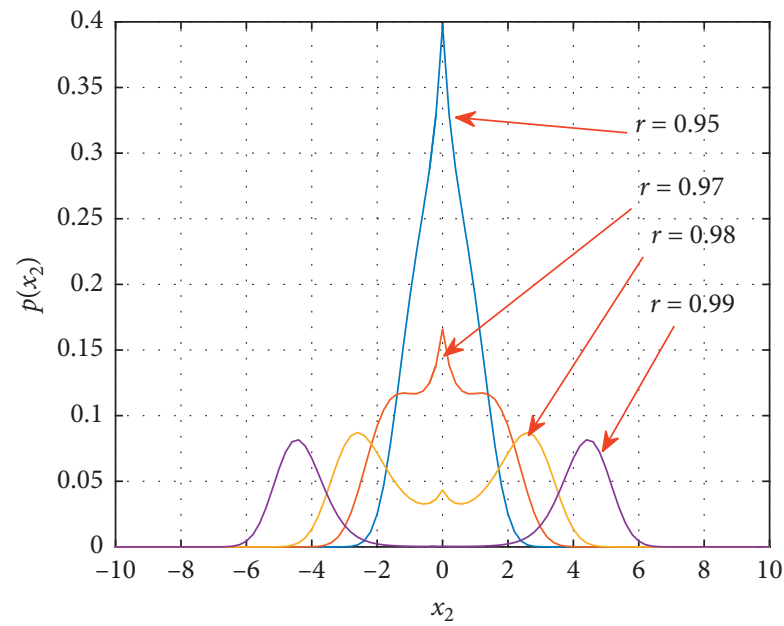

FIgURE 8: Section graphs of joint PDFs on the surface $x_{1}=0$ for different restitution coefficients $r$. 


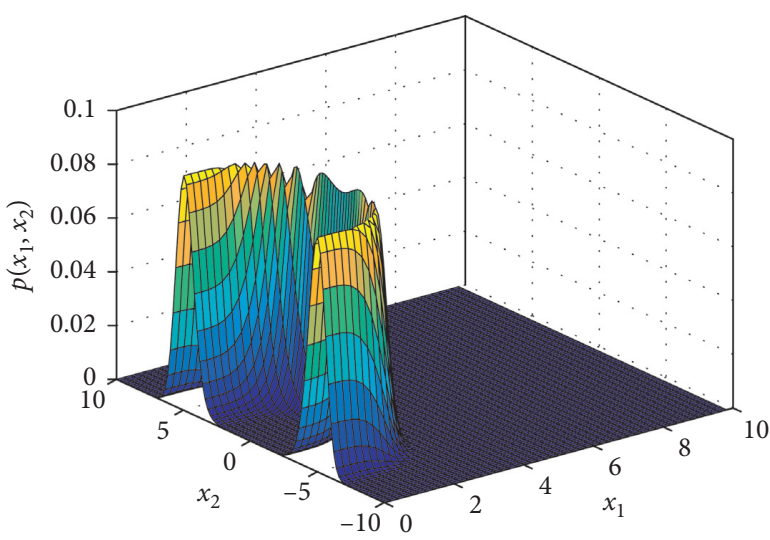

(a)

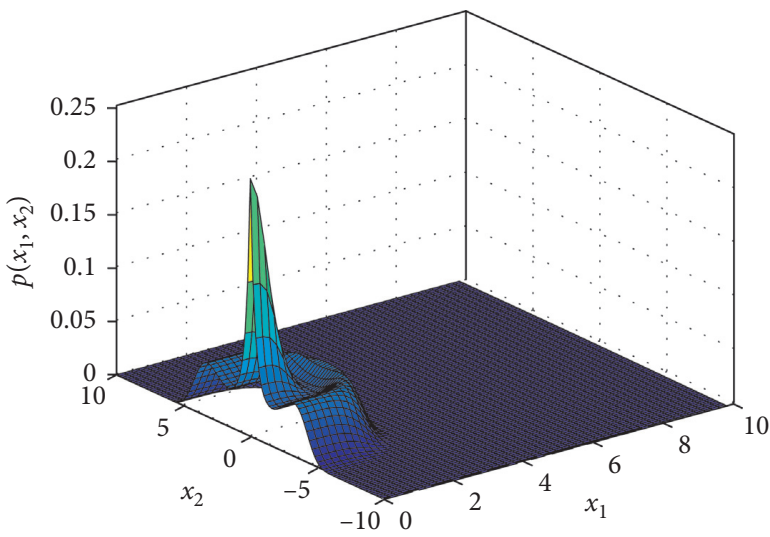

(c)

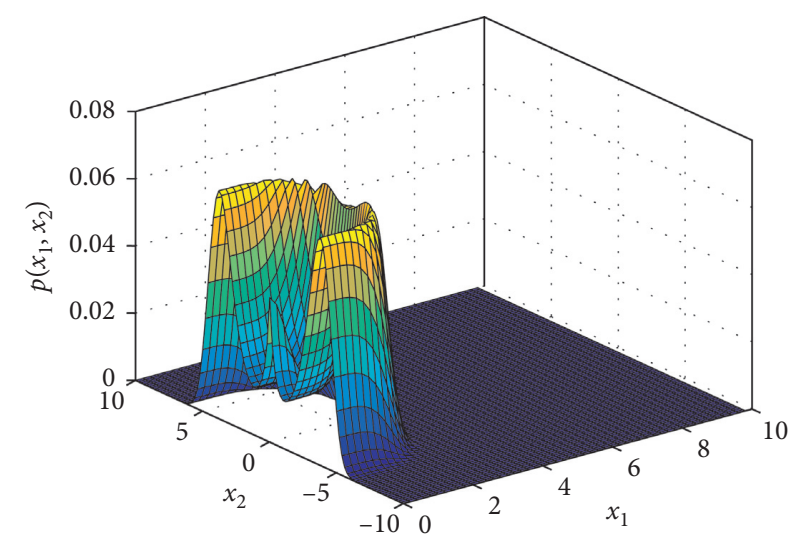

(b)

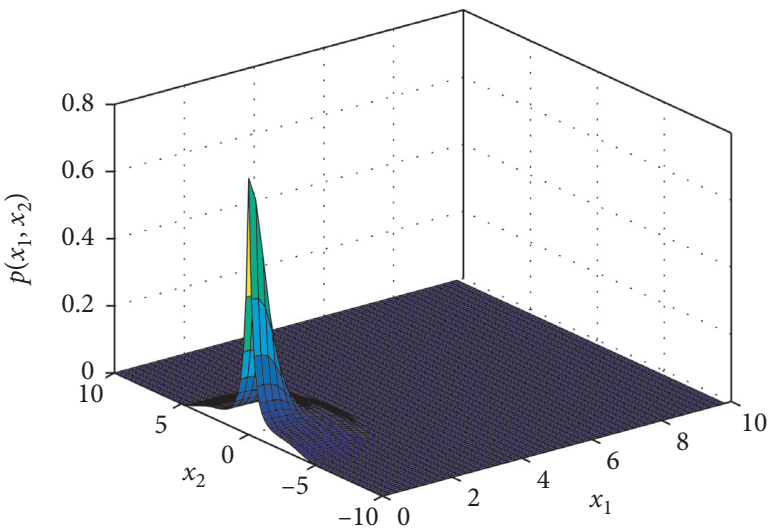

(d)

Figure 9: Analytical results of stationary joint PDFs for displacement $x_{1}$ and velocity $x_{2}$ for different amplitudes of friction: (a) $f_{k}=0$, (b) $f_{k}=2$, (c) $f_{k}=2.5$, and (d) $f_{k}=3$.

amplitudes of friction $f_{k}$ are shown in Figure 10, which clearly present the changes of response PDFs in pace with the variation of $f_{k}$.

4.3. The Influence of Random Excitation. As is known to all, the random excitations are ubiquitous in practical mechanical systems. Their effects are usually not negligible and even play a crucial role. For the sake of understanding the random excitation's effect, the changes of the system's response are studied for different Gaussian white noise intensities. In Figures 11(a) and 11(b), the marginal PDFs for displacement $x_{1}$ and velocity $x_{2}$ are shown, respectively, when $D$ varies; in the meantime, other system parameters are fixed as $c_{1}=3.0, c_{2}=1.0, f_{k}=1.0$, and $r=0.99$. The line denotes the analytical results and the bullet, circle, and asterisk mean the numerical results. It is easy to see that the peak values of marginal PDFs for displacement $x_{1}$ and velocity $x_{2}$ increase with the decrease of noise intensity. However, the shape of the PDFs has no substantial change. That is, there is no stochastic P-bifurcation occurring when the noise intensity changes. The results are similar as other parameters are given. For example, when $c_{1}=3.0, c_{2}=1.0, f_{k}=1.0$, and $r=0.97$, the marginal PDFs for displacement $x_{1}$ and velocity $x_{2}$ are

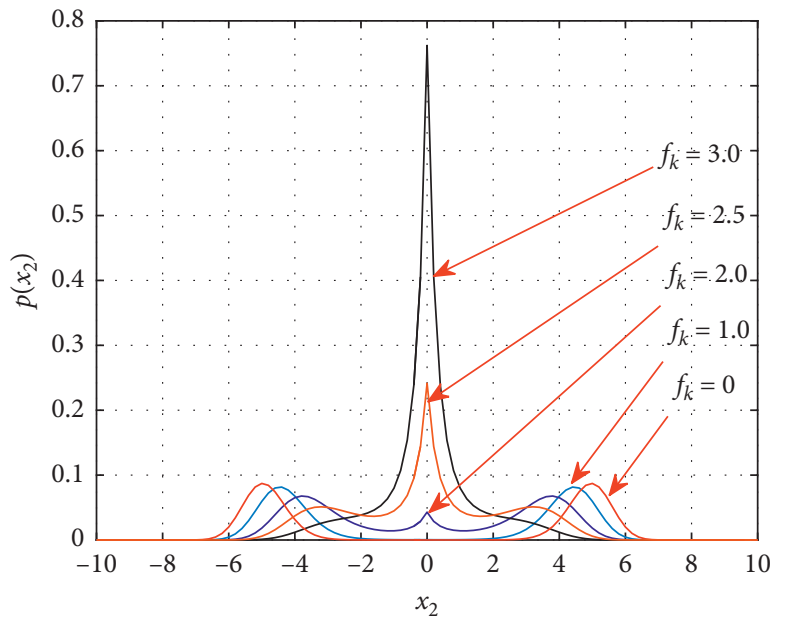

FIGURE 10: Section graphs of joint PDFs on the surface $x_{1}=0$ for different amplitudes of friction $f_{k}$.

shown in Figures 12(a) and 12(b). As can be observed from the figures, the peak values change with the variation of noise intensity, but the shape is little different, which indicates that the stochastic P-bifurcation does not appear. 


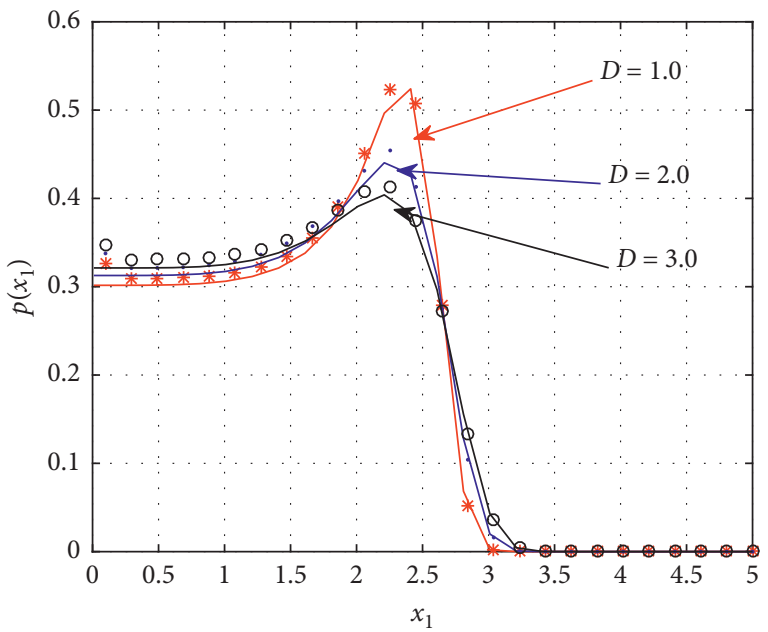

(a)

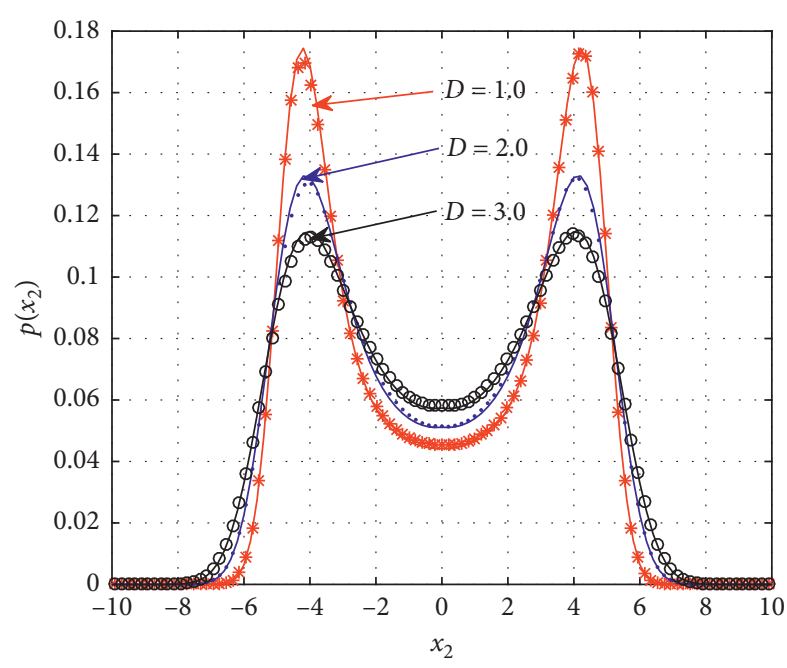

(b)

Figure 11: Stationary PDFs as $r=0.99$ : (a) displacement $x_{1}$; (b) velocity $x_{2}$.

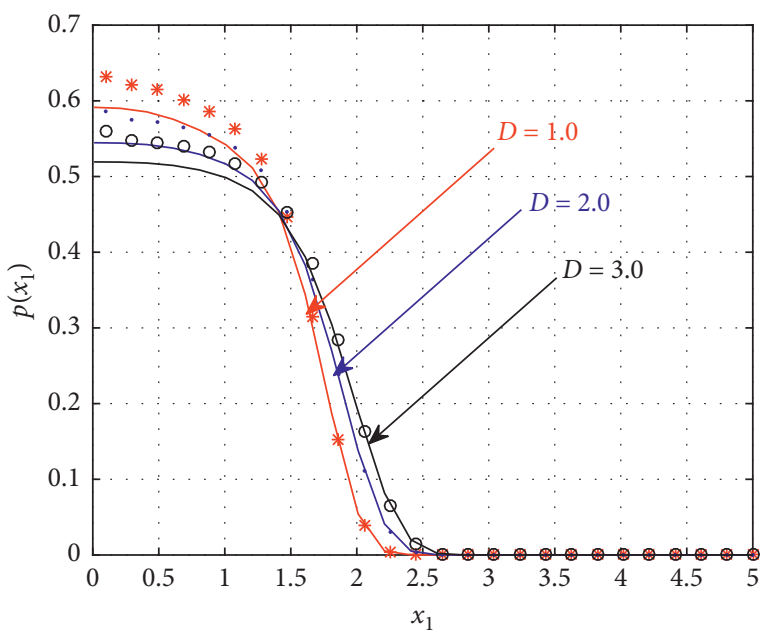

(a)

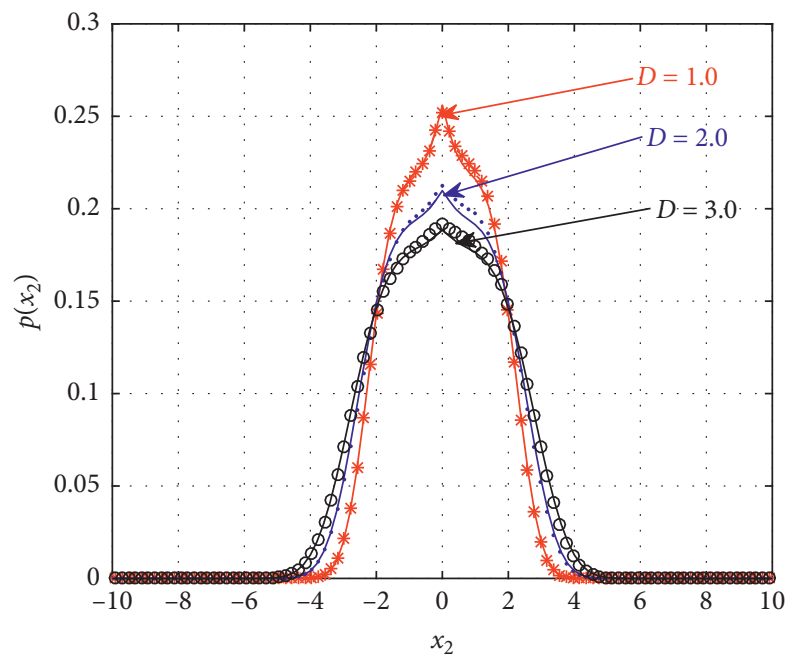

(b)

Figure 12: Stationary PDFs as $r=0.97$ : (a) displacement $x_{1}$; (b) velocity $x_{2}$.

\section{Conclusions}

In this work, the combination of the nonsmooth transformation and the stochastic averaging method of energy envelope is extensively used to obtain the steady-state dynamical responses of a Van der Pol-Duffing vibro-impact system with Coulomb friction subjected to white noise excitation. The results obtained by the analytical procedure agree well with those from Monte Carlo simulation. What's more, effects of different restitution coefficients, amplitudes of friction, and Gaussian white noise intensities on the steady-state dynamical responses are discussed. Results show that the peak values change with the variation of Gaussian white noise intensity and different restitution coefficients and amplitudes of friction can lead to the stochastic P-bifurcation. Generally, the procedure in this paper could be applied to study more complicated nonlinear systems with impact and dry friction.

\section{Data Availability}

The data used to support the findings of this study are included within the article.

\section{Conflicts of Interest}

The authors declare that they have no conflicts of interest.

\section{Acknowledgments}

This work was supported by the National Natural Science Foundation of China (Grant nos. 11702200 and 11702201), Natural Science Basic Research Plan in Shaanxi Province of 
China (Grant no. 2018JQ1088), and Fundamental Research Funds for the Central Universities (Grant no. GHC1911).

\section{References}

[1] W. Zhang and H. Y. Hu, Advance in Nonlinear Dynamics Theory and Application, Science Press, Beijing, China, 2009.

[2] A. Ibrahim Raouf, Vibro-impact Dynamics: Modeling, Mapping and Applications, Springer, Berlin, Germany, 2009.

[3] D. Huang, S. Zhou, and G. Litak, "Analytical analysis of the vibrational tristable energy harvester with a $\mathrm{RL}$ resonant circuit," Nonlinear Dynamics, vol. 97, no. 1, pp. 663-677, 2019.

[4] D. Huang, S. Zhou, and G. Litak, "Theoretical analysis of multi-stable energy harvesters with high-order stiffness terms," Communications in Nonlinear Science and Numerical Simulation, vol. 69, pp. 270-286, 2019.

[5] M. F. Dimentberg, Statistical Dynamics of Nonlinear and Time-Varying Systems, Wiley, London, UK, 1988.

[6] D. P. Jin and H. Y. Hu, "Vibroimpacts and their typical behaviors of mechanical systems," Advances in Mechanics, vol. 25, no. 2, pp. 155-164, 1999.

[7] K. Wang and X. B. Liu, "Stochastic resonance for a timedelayed metapopulation system driven by multiplicative and additive noise," Chinese Physics Letters, vol. 20, no. 7, p. 70504 , 2013.

[8] Y. Xu, Y. Li, and D. Liu, "A method to stochastic dynamical systems with strong nonlinearity and fractional damping," Nonlinear Dynamics, vol. 83, no. 4, pp. 1-11, 2015.

[9] G. Q. Cai and Y. K. Lin, "Response distribution of non-linear systems excited by non-Gaussian impulsive noise," International Journal of Non-linear Mechanics, vol. 27, no. 6, pp. 955-967, 1992.

[10] M. F. Dimentberg and D. V. Iourtchenko, "Random vibrations with impacts: a review," Nonlinear Dynamics, vol. 36, no. 2-4, pp. 229-254, 2004.

[11] N. S. Namachchivaya and J. H. Park, "Stochastic dynamics of impact oscillators," Journal of Applied Mechanics, vol. 72, no. 6, pp. 862-870, 2005.

[12] K. Kappaganthu and C. Nataraj, "Nonlinear modeling and analysis of a rolling element bearing with a clearance," Communications in Nonlinear Science and Numerical Simulation, vol. 16, no. 10, pp. 4134-4145, 2011.

[13] Q. Ding and H. M. Zhai, "Progress in friction dynamics of mechanical systems," Advance in Mechanics, vol. 43, no. 1, pp. 112-131, 2013.

[14] G. Luo, Y. Zhang, J. Xie, and J. Zhang, "Periodic-impact motions and bifurcations of vibro-impact systems near 1:4 strong resonance point," Communications in Nonlinear Science and Numerical Simulation, vol. 13, no. 5, pp. 1002-1014, 2008.

[15] S. W. Shaw and P. J. Holmes, "A periodically forced piecewise linear oscillator," Journal of Sound and Vibration, vol. 90, no. 1, pp. 129-155, 1983.

[16] G.-W. Luo and J.-H. Xie, "Hopf bifurcation of a two-degreeof-freedom vibro-impact system," Journal of Sound and Vibration, vol. 213, no. 3, pp. 391-408, 1998.

[17] J. M. T. Thompson and R. Ghaffari, "Chaos after perioddoubling bifurcations in the resonance of an impact oscillator," Physics Letters A, vol. 91, no. 1, pp. 5-8, 1982.

[18] A. B. Nordmark, "Existence of periodic orbits in grazing bifurcations of impacting mechanical oscillators," Nonlinearity, vol. 14, no. 6, pp. 1517-1542, 2001.
[19] A. B. Nordmark and P. T. Piiroinen, "Simulation and stability analysis of impacting systems with complete chattering," Nonlinear Dynamics, vol. 58, no. 1-2, pp. 85-106, 2009.

[20] K. M. Cone and R. I. Zadoks, "A numerical study of an impact oscillator with the addition of dry friction," Journal of Sound and Vibration, vol. 188, no. 5, pp. 659-683, 1995.

[21] Q. Feng and H. He, "Modeling of the mean Poincaré map on a class of random impact oscillators," European Journal of Mechanics-A/Solids, vol. 22, no. 2, pp. 267-281, 2003.

[22] J. Q. Sun, "Random vibration analysis of a non-linear system with dry friction damping by the short-time Gaussian cell mapping method," Journal of Sound and Vibration, vol. 180, no. 5, pp. 785-795, 1995.

[23] L. N. Virgin and C. J. Begley, "Grazing bifurcations and basins of attraction in an impact-friction oscillator," Physica D, vol. 132, pp. 43-57, 2001.

[24] Y. Q. Zhang, W. C. Ding, and C. Sun, "Bifurcation and chaos of a vibration system with clearance and dry friction," $V i$ bration and Shock, vol. 27, no. 7, pp. 102-105, 2008.

[25] Y. Yue, P. Miao, and J. Xie, "Coexistence of strange nonchaotic attractors and a special mixed attractor caused by a new intermittency in a periodically driven vibro-impact system," Nonlinear Dynamics, vol. 87, no. 2, pp. 1187-1207, 2017.

[26] H.-S. Jing and K.-C. Sheu, "Exact stationary solutions of the random response of a single-degree-of-freedom vibro-impact system," Journal of Sound and Vibration, vol. 141, no. 3, pp. 363-373, 1990.

[27] Z. L. Huang, Z. H. Liu, and W. Q. Zhu, "Stationary response of multi-degree-of-freedom vibro-impact systems under white noise excitations," Journal of Sound and Vibration, vol. 275, no. 1-2, pp. 223-240, 2004.

[28] M. Xu, Y. Wang, X. L. Jin, Z. L. Huang, and T. X. Yu, "Random response of vibro-impact systems with inelastic contact," International Journal of Non-linear Mechanics, vol. 52, pp. 26-31, 2013.

[29] V. F. Zhuravlev, "A method for analyzing vibration-impact systems by means of special function," Mechanical Solids, vol. 11, pp. 23-27, 1976.

[30] M. F. Dimentberg and D. V. Iourtchenko, "Towards incorporating impact losses into random vibration analyses: a model problem," Probabilistic Engineering Mechanics, vol. 14, no. 4, pp. 323-328, 1999.

[31] M. F. Dimentberg and A. I. Menyailov, "Response of a singlemass vibroimpact system to white-noise random excitation," ZAMM - Zeitschrift für Angewandte Mathematik und Mechanik, vol. 59, no. 12, pp. 709-716, 1979.

[32] D. V. Iourtchenko and L. L. Song, "Analytical analysis of stochastic vibroimpact systems," in Proceedings of International Conference on Structural Safety and Reliability 2005, pp. 1931-1937, Rome, Italy, June 2005.

[33] H. T. Zhu, "Response of a vibro-impact Duffing system with a randomly varying damping termffng system with a randomly varying damping term," International Journal of Non-linear Mechanics, vol. 65, pp. 53-62, 2014.

[34] L. Chen, J. Qian, H. Zhu, and J.-Q. Sun, “The closed-form stationary probability distribution of the stochastically excited vibro-impact oscillators," Journal of Sound and Vibration, vol. 439, pp. 260-270, 2019.

[35] X. Xie, J. Li, D. Liu, and R. Guo, "Transient response of nonlinear vibro-impact system under Gaussian white noise excitation through complex fractional moments," Acta Mechanica, vol. 228, no. 3, pp. 1153-1163, 2017. 
[36] W. J. Ding, Damping Theory, Tsinghua University Press, Beijing, China, 1988.

[37] J. Sun, W. Xu, and Z. Lin, "Research on the reliability of friction system under combined additive and multiplicative random excitations," Communications in Nonlinear Science and Numerical Simulation, vol. 54, pp. 1-12, 2018.

[38] M. Su, W. Xu, and G. Yang, "Response analysis of van der Pol vibro-impact system with Coulomb friction under Gaussian white noise," International Journal of Bifurcation and Chaos, vol. 28, no. 13, Article ID 1830043, 2018.

[39] R. Z. Khasminskii, "On the averaging principle for stochastic differential Ito equation," Kibernetika, vol. 4, no. 3, pp. 260279, 1968.

[40] R. L. Stratonovich, Topics in the Theory of Random Noise, Gordon and Gordon, New York, NY, USA, 1967.

[41] W. Zhu and Y. K. Lin, "Stochastic averaging of energy envelope," Journal of Engineering Mechanics, vol. 117, no. 8, pp. 1890-1905, 1991. 